\title{
PULSED $\mathrm{CO}_{2}$-LASER EXCITATION OF $\mathrm{O}_{3} / \mathrm{O}_{2}$ MIXTURES AT PRESSURES FROM 0.16 TO 1.20 BAR
}

\author{
B. RAFFEL $\dagger$ and J. WOLFRUM \\ Physikalisch-Chemisches Institut, Ruprecht-Karls-Universität, Im \\ Neuenheimer Feld 253, D-6900 Heidelberg, Federal Republic of Germany
}

(Received November 21, 1989)

\begin{abstract}
An investigation is presented of the transient vibrational excitation of $\mathrm{O}_{3}$ in the collision dominated regime initiated by pulsed $\mathrm{CO}_{2}$-laser radiation. IR-UV-double resonance experiments and measurements of the absorbance for the $\mathrm{CO}_{2}$-laser lines 9P18, 20, and 22 were carried out. Mixtures of $\mathrm{O}_{3}(p=17 \mathrm{mbar})$ with $\mathrm{O}_{2}$ were investigated at pressures of $160 \mathrm{mbar} \leq p_{\text {mixt }} \leq 1200 \mathrm{mbar}$ and laser fluences of $0.01 \mathrm{~J} / \mathrm{cm}^{2} \leq$ $F_{\text {in }}<2 \mathrm{~J} / \mathrm{cm}^{2}$. The results are interpreted by numerical simulations in terms of a comprehensive excitation/relaxation model based upon SSH-theory. Concerning the evolution of the excitation, simulated transients of the UV-absorbance compare well with the corresponding observed signals. The saturation of the absorbing $\mathrm{O}_{3}$-transitions is demonstrated by the measured fluence dependence of the absorption coefficient at the laser wavelengths. The extent of the $\mathrm{O}_{3}$-excitation can be deduced according to the model from the maximum vibrational temperature $T_{m}$ reached in the $v_{1}$ - and $v_{3}$-oscillators. $T_{m}$ is accessible via the UV-transients and also via the absorbed laser energy in the case of slow relaxation at 160 mbar $\leq p_{\text {mixt }} \leq 340 \mathrm{mbar}$. In this range both techniques result in the same values for $T_{m}$. The experimental and the corresponding simulated $T_{m}$ depend exponentially on the laser fluence $\left(T_{m}=\right.$ const $\left.\cdot \bar{F}_{\text {in }}{ }^{0.3}\right)$ provided $T_{m}>400 \mathrm{~K}$ being also confirmed up to $p_{\text {mixt }}=1200 \mathrm{mbar}$ by the observed UV-transients.
\end{abstract}

\section{INTRODUCTION}

This presentation is part of a series of publications on the laser ignition of $\mathrm{O}_{2} / \mathrm{O}_{3}$ mixtures. ${ }^{1-7}$ Such mixtures can easily be ignited by $\mathrm{CO}_{2}$-lasers and show a simple decomposition chemistry with limited number of elementary steps and are therefore very suitable to detailed numerical simulation techniques. ${ }^{5,8}$ In many other cases, for example in the classical spark ignition, the knowledge on the totality of processes affecting the conversion of the external energy into a moving flame is still incomplete. Therefore one is interested in the creation of a completely definable experimental situation potentially given by controlled laser excitation.

In the earlier investigations ${ }^{6,7}$ we proved that as a standard system for the numerical simulation of flame phenomena, ${ }^{9,10}$ the ozone decomposition flame $\left(\mathrm{O}_{2} / \mathrm{O}_{3}\right.$-flame $)$, can be ignited by a $\mathrm{CO}_{2}$-laser pulse in a reproducible way. The experiments were carried out in a cylindrical cell irradiating the axial region with the laser pulse from one side. The resulting flame front was observed to be conical in shape. The radial component of the flame propagation proved to depend only on the

$\dagger$ Permanent address: Bayer AG, Abt. FS-IT, D-4047 Dormagen. 
mixture composition, the longitudinal component, however, depended also on the laser fluence because of the longitudinal gradient of laser excitation. This gradient is in turn governed by the spatial and temporal course of the molecular excitation and relaxation during and after the irradiation. The characterization of the energy exchange between the external light field and the various degrees of freedom of the gas mixture is therefore essential to describe the resulting onset of the chemical reactions and furthermore, the observed macroscopic flame phenomena.

In this publication we present experimental data for the basic excitation/relaxation processes in non-ignitable $\mathrm{O}_{2} / \mathrm{O}_{3}$-mixtures. To meet, but also to extend the conditions in the ignition experiments, gas mixtures were investigated at pressures up to $p_{\text {mixt }}=1200$ mbar, having been at room temperature before the laser irradiation.

In addition, this range of conditions is of general interest for the dynamics of vibrational excitations since the fast, high-power laser pumping coincides with a very efficient molecular relaxation. A practicable but nevertheless comprehensive model based upon the relaxation theory of harmonic oscillators ( $\mathrm{SSH}$ theory) ${ }^{\mathbf{1 1}}$ is sufficient to describe the features of these processes given by the experimental findings. In detail, the temporal course of the transient vibrational excitation for various total pressures as well as the fluence dependence of the maximum excitation are reproduced correctly.

The technique of the ignition experiments ${ }^{1-7}$ can also be used for the present investigation, i.e. probing of the absorbed laser energy and of the UV-transmittance in the $\mathrm{O}_{3}$-Hartley-continuum at right angles to the laser irradiation. A short recapitulation of the experiment will be given in ch. 2 .

The results are interpreted quantitatively following the simplified model for the vibrational relaxation, ${ }^{11}$ adapted to ozone by Rosen and Cool. ${ }^{12}$ The flow of the internal energy between the various degrees of freedom as well as the propagation of the laser light in the absorbing medium can be described by a system of differential equations as given in ch. 3. Depending on the experimental conditions, several features must be accounted for, such as the high resolution ozone spectrum, the pressure broadening of the spectral lines, the saturation of the ro-vibrational transitions, and the rotational bottleneck.

The system of differential equations can be simulated numerically, leading to results that are directly comparable with experimental findings. As an example, experimental and simulated UV-transients are presented in ch. 4 .

In the last chapter, experimental evidence is given for the saturation of the ozone transitions via the fluence dependence of the absorption coefficient measured at various pressures of the gas mixture up to $p_{\text {mixt }}=1200$ mbar. The excitation of the laser pumped $\mathrm{O}_{3}$-oscillator can be described by the maximum vibrational temperature reached in this oscillator being accessible by means of the measured ozone $\mathrm{UV}$-absorbance. Due to saturation effects this temperature is observed to depend exponentially on the laser fluence, not only in the experiments but also in the simulation according to the relaxation model.

Throughout these chapters the quality and the significance of various parameters from the literature (spectral data, relaxation constants) for the correct simulation of an excitation/relaxation process and therefore the laser ignition will be discussed. 


\section{EXPERIMENTAL}

The investigation of the excitation and relaxation processes was carried out in the experimental arrangement of the ignition experiments ${ }^{4,6}$ outlined in Figure 1. Briefly, the pulsed light of a line tuned, multi-mode TEA- $\mathrm{CO}_{2}$-laser is slightly focused along the axis of a cylindrical cell. The cell consists of a quartz cylinder of 64 $\mathrm{mm}$ length and $18 \mathrm{~mm}$ diameter fitted at both ends with $\mathrm{KCl}$ windows. The 1/e-diameter of the laser beam waist amounts to $2.7 \mathrm{~mm}$, being nearly constant along the whole cell length. ${ }^{6,7}$ The shape of the radial profile varies considerably because of the imaging. In the image plane at half of the cell length, the profile is almost rectangular and is given approximately by a Gaussian expression for the laser fluence

$$
F(r) \sim \exp \left[-(r / a)^{n}\right]
$$

where $r$ is the radius, $a$ the $1 / \mathrm{e}$-radius and $n=32$. For increasing distance from this plane, the profile levels off and $n$ reduces to roughly $n=4$ at the cell windows. ${ }^{6,7}$ On a small scale, local structures are superimposed on the smooth profile (2.1) and these vary from shot to shot because of the interference pattern of the laser modes. ${ }^{7}$ In the time domain mode, beating thus leads to a partly oscillatory behaviour of the laser intensity. ${ }^{7}$ The laser pulse as a whole consists of a leading spike of approximately 0.15 $\mu$ sec half-width (FWHM) followed by a decaying tail of about $1 \mu$ sec half-width (c.f. Figure 1 in $^{6}$ ), varying slightly with the nitrogen content of the laser gas mixture.

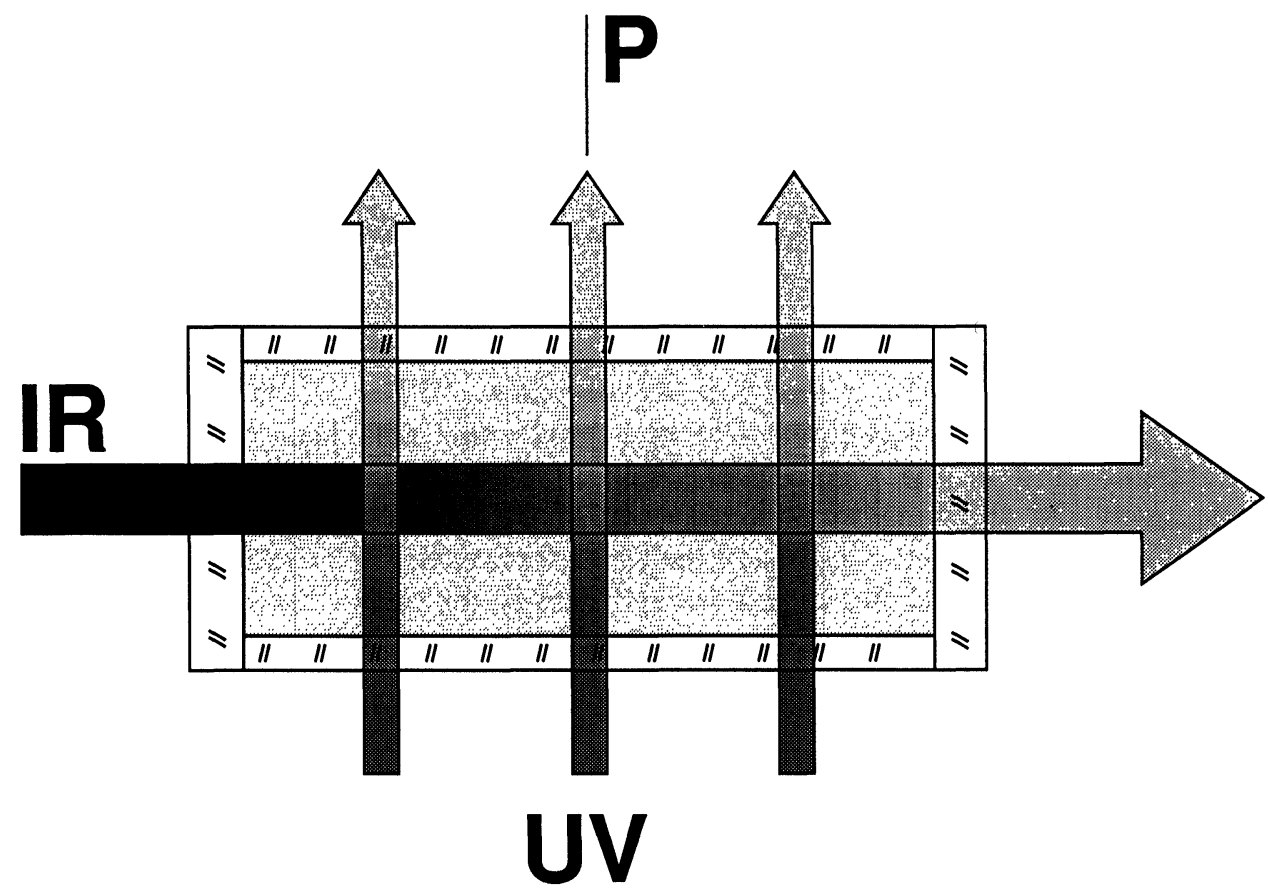

Figure 1 Experimental arrangement for the IR-UV-double resonance experiments; cell length $=64$ $\mathrm{mm}$, cell diameter $=18 \mathrm{~mm}, P=$ image plane of the laser telescope. 
The excitation experiments were carried out with non-ignitable $\mathrm{O}_{2} / \mathrm{O}_{3}$-mixtures. Throughout this investigation the ozone pressure was kept at $p_{\mathrm{O}_{3}}=17 \mathrm{mbar}$ in mixtures of $160,340,690$, and 1200 mbar total pressure, respectively. Therefore, the concentration of oxygen is in all cases $>90 \%$ ensuring only one predominant kind of collision partner for the ozone molecules.

The excitation processes were investigated by two independent techniques. ${ }^{7}$ First, the incoming and outgoing pulse energy was measured by pyroelectric detectors (gen tec ED 100) leading to the mean absorbed energy density in the irradiated volume that is given approximately by the cell length and the 1/e-radius of the laser beam. Additionally, the absorption coefficient at the laser wavelength under investigation, the number of absorbed laser photons per ozone molecule, and the maximum vibrational temperature in the ozone- $\left(v_{1}, v_{3}\right)$-oscillators can be calculated.

The second method monitors the UV-absorbance in the ozone Hartley-continuum which depends strongly on the molecular excitation especially in its long wavelength wing. In the case of thermal excitation, the temperature dependence of the absorption coefficient is concentrated in a modified Sulzer-Wieland expression for $210 \mathrm{~nm}$ $\leq \lambda \leq 320 \mathrm{~nm}$ and $300 \mathrm{~K} \leq T \leq 1050 \mathrm{~K}^{13}$ :

$$
\begin{aligned}
& \alpha(v, T)=\alpha_{a 0}\left[\tanh \left(\frac{\Theta_{a e}}{2 T}\right)\right]^{1 / 2} \exp \left[-\tanh \left(\frac{\Theta_{a e}}{2 T}\right)\left(\frac{v-v_{a}}{\Delta v_{a 0}}\right)^{2}\right] \\
&+\alpha_{b 0} \exp \left[-\frac{T}{\Theta_{b e}}-\left(\frac{v-v_{b 0}}{\Delta \alpha_{b 0}}\right)^{2}\right] \\
& \text { with } \alpha_{a 0}=126.14 \mathrm{~atm}^{-1} \mathrm{~cm}^{-1} \\
& v_{a 0}=38800 \mathrm{~cm}^{-1} \Theta_{a e}=900 \mathrm{~K} \\
& \alpha_{b 0}=66.02 \mathrm{~atm}^{-1} \mathrm{~cm}^{-1}=2900 \mathrm{~cm}^{-1} \\
& v_{b 0}=43000 \mathrm{~cm}^{-1} \\
& \Theta_{b e}=660 \mathrm{~K} \\
& \Delta v_{b 0}=3300 \mathrm{~cm}^{-1}
\end{aligned}
$$

The variation of the UV-transmittance was observed on three probe beams at right angles to the laser beam, as in the ignition experiments. ${ }^{4,6}$ The beams were located in the image plane at half way along cell length, where the radial laser profile is almost rectangular, and at the distance of $2 \mathrm{~cm}$ in front of and behind this plane, respectively. At the long wavelength wing of the Hartley-continuum, the laser excitation leads to a steep decrease of the transmittance (c.f. Figure 5) followed by a slower increase to the former value corresponding to the relaxation of the molecular excitation. The transmitted light was collected by photomultipliers (1P28 a.o.); the corresponding signals were amplified and recorded by a transient-recorder (Krenz TRC 6070). The upper fall-off $(-3 \mathrm{~dB})$ of this arrangement was determined at about $20 \mathrm{MHz}$.

The shape of the transient UV-signals was greatly simplified compared to that in the ignition experiments since the laser heating of the low-concentration mixtures was not sufficient to effect an observable reduction of the signal remaining after the relaxation process. Likewise, no super-imposed oscillatory components due to 
acoustic oscillations of the cylindrical gas volume could be observed on the transients. Furthermore, the minor absorption of the laser light in these gas mixtures led to a homogeneous excitation along the cell length and consequently to roughly identical transient signals on the three beams. The use of the average minimum level of the three transients for a quantitative representation of the laser excitation is therefore justified. It corresponds to an elevated absorption coefficient $\alpha_{T}$ within the volume of intersection of the laser and the probe beams:

$$
\alpha_{T}=\alpha_{0}+\frac{1}{2 a p_{\mathrm{O}_{3}}} \log \frac{I_{0}}{I}
$$

where $\alpha_{0}$ is the absorption coefficient before the irradiation, $p_{\mathrm{O}_{3}}$ the ozone pressure, $a$ the $1 / \mathrm{e}$-radius of the laser beam, $I_{0}$ the UV-intensity before the irradiation, and $I$ the minimum intensity. By means of expression (2.2) $\alpha_{T}$ can be assigned to a temperature $T$ which is significant for the maximum molecular excitation.

\section{THEORY}

\subsection{The Excitation/Relaxation Model}

The straightforward relaxation model of Rosen and $\mathrm{Cool}^{12}$ proved to be adequate to account for the findings of the present investigation. The ozone molecule is looked upon as a system of two coupled harmonic oscillators $\left(v_{a}, v_{b}\right)$ and can therefore be reduced to the kinetic expressions of Schwartz, Slawsky and Hertzfeld. ${ }^{11}$ One of the oscillators is two-fold degenerate with a vibrational quantum of $v_{a}=\left(v_{1}+v_{3}\right) / 2$ representing the approximately equi-energetic symmetric $\left(v_{1}=1103.157 \mathrm{~cm}^{-114}\right)$ and asymmetric $\left(v_{3}=1042.096 \mathrm{~cm}^{-114}\right)$ stretching modes of the real ozone molecule. This replacement is possible since there is a fast exchange according to

$$
\mathrm{O}_{3}(001)+M \longrightarrow \mathrm{O}_{3}(100)+M, M=\mathrm{O}_{3}
$$

The rate of this reaction is higher than $1.2 \cdot 10^{5} \mathrm{mbar}^{-1} \mathrm{sec}^{-112}$ and presumably given by the gas kinetic collision frequency, ${ }^{15}$ i.e. $3.3 \cdot 10^{6} \mathrm{mbar}^{-1} \mathrm{sec}^{-1}$ at $T=300$ $\mathrm{K}$. For an ozone pressure of $17 \mathrm{mbar}$, as in this investigation, the exchange reaction (3.1) takes $18 \mathrm{nsec}$, i.e. much faster than the laser pulse. Moreover, a further acceleration is effected by the oxygen component, mainly due to its abundance, although the rate of (3.1) should be less in case of $M=\mathrm{O}_{2}{ }^{16}$ because of missing resonance effects. Thus after pumping the asymmetric stretch $v_{3}$, the absorbed energy is expected to equilibrate almost instantaneously with the $v_{1}$-mode. The intramode equilibration in the $v_{3}$-mode should also take place at nearly gas kinetic collision frequency. With the lack of direct data this can at least be adopted by comparison with detailed results for other triatomic gases, e.g. $\mathrm{CO}_{2} \cdot{ }^{17}$ Therefore, the concept of the $v_{a}$-oscillator being in permanent but time varying vibrational equilibrium throughout the laser pumping and relaxation processes is justified. The energy loss of this oscillator is governed by the energy gap law for vibrational relaxation ${ }^{18}$ in the sense that the intermode relaxation to the $v_{b}$-oscillator that corresponds to the bending mode of $\mathrm{O}_{3}\left(v_{2}=701.42 \mathrm{~cm}^{-114}\right)$ is more probable than 


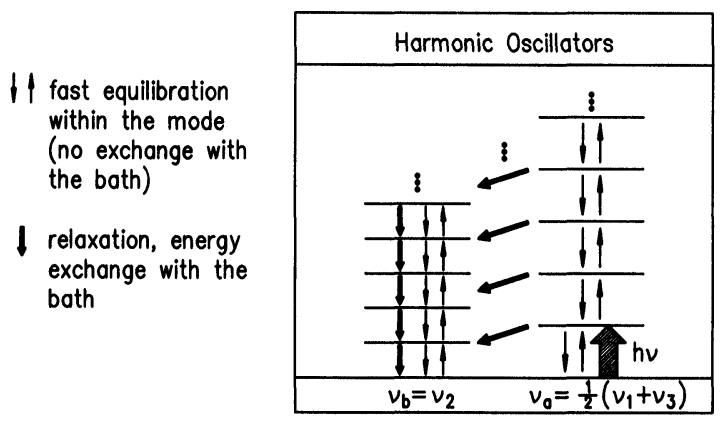

Figure 2 The model for the laser excitation and relaxation in the ozone molecules.

the $V-T$ relaxation within the $v_{a}$-oscillator c.f. Figure 2 . The intermode exchange is followed by the $V-T$ relaxation within the $v_{b}$-oscillator explaining therefore the observation of bi-exponential signals in. ${ }^{12}$ To complete the general description of the model it should be mentioned that molecular dissociation and also transport processes are not measurable after the laser shot. ${ }^{7}$ Therefore, these processes are neglected in this investigation.

The relaxation of the molecular excitation can be described by a system of kinetic equations for the energy content of the $v_{a^{-}}$and $v_{b}$-oscillator and the remaining degrees of freedom, respectively. Within this framework light absorption can be accounted for simply by the addition of an appropriate term. According to ${ }^{19}$ it is justified to discard a more elaborate coherent description since the multi-mode laser light (spectral width $\Delta v \approx 0.03 \mathrm{~cm}^{-1}$ ) leads to a coherence time $\Delta t \sim 1 /(4 \pi c \Delta v)^{20}$ being shorter than the period of the Rabi-oscillations.

In the system of rate equations only one spatial dimension-the longitudinal coordinate $z$-is retained, for simplicity. It allows the evaluation of the spread of the laser light as well as the pumping and relaxation processes along the cell axis. The evolution of the spatial density $E_{a}(z, t)$ of the vibrational energy in the $v_{a}$-oscillator is determined by the rate of light absorption that is proportional to the energy density $w(z, t)$ of the laser light and by the rate $k_{a b}$ of relaxation to the $v_{b}$-oscillator:

$$
\frac{\partial E_{a}}{\partial t}=-k_{a b} \cdot R_{a b}+A \cdot w
$$

The corresponding equation for the spatial energy density $E_{b}(z, t)$ of the $v_{b}$-oscillator describes the competing processes of the intermode relaxation and the $V-T$ relaxation in the $v_{b}$-oscillator (rate $k_{b}$ ):

$$
\frac{\partial E_{b}}{\partial t}=-k_{b} \cdot R_{b}+\frac{v_{b}}{v_{a}} k_{a b} \cdot R_{a b}
$$

The term $v_{b} / v_{a}$ reflects that only this portion of the $v_{a}$-quantum is transferred to the $v_{b}$-oscillator by relaxation whereas the exceeding portion as well as the $V-T$ 
relaxation in the $v_{b}$-oscillator increase the temperature $T(z, t)$ of the bath:

$$
\frac{\partial T}{\partial t}=\left\{\left(1-\frac{v_{b}}{v_{a}}\right) k_{a b} R_{a b}+k_{b} R_{b}\right\} / C
$$

The bath consists of the rotational and translational ozone degrees of freedom and the totality of the oxygen degrees of freedom. Therefore, the corresponding heat capacity $C$ is given by

$$
C=3 \frac{p_{\mathrm{O}_{3}}}{T_{0}}+\frac{c_{v, \mathrm{O}_{2}}(T)}{R} \cdot \frac{p_{\mathrm{O}_{2}}}{T_{0}}
$$

with $T_{0}$ the room temperature, and $p_{\mathrm{O}_{3}}$ and $p_{\mathrm{O}_{2}}$ the partial pressures of the ozone and oxygen component, respectively. The temperature dependent contribution of the oxygen $c_{v, \mathrm{O}_{2}}(T) / R$ is given by an empirical polynomial expression. ${ }^{10,21}$ The relaxation constants $k_{a b}$ and $k_{b}$ can be summed from contributions of the various collision partners: ${ }^{22,23}$

$$
\begin{aligned}
& k_{a b}=p_{\mathrm{O}_{2}} \cdot k_{a b, \mathrm{O}_{2}}+p_{\mathrm{O}_{3}} \cdot k_{a b, \mathrm{O}_{3}} \\
& k_{b}=p_{\mathrm{O}_{2}} \cdot k_{b, \mathrm{O}_{2}}+p_{\mathrm{O}_{3}} \cdot k_{b, \mathrm{O}_{3}}
\end{aligned}
$$

The individual constants given by several authors still varying by more than a factor of 2 , c.f. the compilation in ${ }^{24}$. The best set of data matching this compilation and also the findings of the present investigation is

$$
\begin{aligned}
k_{a b, \mathrm{O}_{2}} & =698 \mathrm{mbar}^{-1} \mathrm{sec}^{-1} \\
k_{b, \mathrm{O}_{2}} & =2115 \mathrm{mbar}^{-1} \mathrm{sec}^{-1} \\
k_{a b, \mathrm{O}_{3}} & =6450 \mathrm{mbar}^{-1} \mathrm{sec}^{-1} \\
k_{b, \mathrm{O}_{3}} & =2145 \mathrm{mbar}^{-1} \mathrm{sec}^{-1} .
\end{aligned}
$$

The amount $R_{a b}$ and $R_{b}$ of energy density transferred by relaxation is governed by the actual energy content $E_{a}$ and $E_{b}$ of the $v_{a}$-and the $v_{b}$-oscillator and by the energy content $E_{a}(T)$ and $E_{b}(T)$ the oscillators would have at the actual equilibrium temperature $T(z, t)$ of the bath (c.f. $\left.{ }^{11,12}\right)$ :

$$
\begin{gathered}
R_{a b}=\frac{1}{2} E_{a} \frac{E_{b}}{E_{b}(T)} \cdot \\
\left\{\left(1-\frac{E_{a}(T)}{E_{a}}\right) \cdot \frac{1-\exp \left[-\frac{h c v_{a}}{k T}\right]}{1-\exp \left[-\frac{h c v_{b}}{k T}\right]}-\left(1-\frac{E_{b}(T)}{E_{b}}\right) \cdot\left(1-\exp \left[-\frac{h c v_{b}}{k T}\right]\right)\right\} \\
R_{b}=\left[E_{b}-E_{b}(T)\right] \cdot\left(1-\exp \left[-\frac{h c v_{b}}{k T}\right]\right) .
\end{gathered}
$$


$E_{a}(T)$ and $E_{b}(T)$ are given by the well-known Einstein function for the energy of a harmonic oscillator at the temperature $T:^{11,12}$

$$
E_{a}(T)=\frac{2 N_{\mathrm{O}_{3}} h c v_{a}}{\exp \left[\frac{h c v_{a}}{k T}\right]-1}, E_{b}(T)=\frac{N_{\mathrm{O}_{3}} h c v_{b}}{\exp \left[\frac{h c v_{b}}{k T}\right]-1},
$$

with $N_{\mathrm{O}_{3}}$ the number density of the ozone molecules, $h$ Planck's constant, $k$ Boltzmann's constant, and $c$ the velocity of light. The spread of the energy density $w$ of the laser light along the $z$-direction can be described by the continuity equation ${ }^{25}$

$$
\frac{\partial w}{\partial t}+c \cdot \frac{\partial w}{\partial z}=-A \cdot w
$$

The rate of absorption $A$ depends in a complicated way on the time dependent local conditions. As a boundary condition, the energy density $w$ of the laser light at the entrance window $(z=0)$ is determined from the total incoming pulse energy $E_{\text {in }}$ using the expression

$$
w(0, t)=\frac{1}{c} \cdot \frac{E_{\text {in }}}{S} \cdot f(t), \quad \int_{-\infty}^{\infty} f(t) \mathrm{d} t=1
$$

For this investigation it is sufficient to relate $w$ to the cross section $S=\pi a^{2}$ which is given by the $1 / e$-radius $a$ of the beam profile. The normalized function $f(t)$ represents the temporal pulse profile observed with a photon drag detector. ${ }^{1,6}$ Its global evolution can be approximated by a polygonal line, for simplicity.

\subsection{The Absorption Term}

The energy density $A \cdot w$ of laser light absorbed at position $z$ and time $t$ depends on the spectroscopic features of the gas mixture, on the actual occupation of the ro-vibrational levels of the ozone molecules, and on the locally available light density $w$ :

$$
A \cdot w=c \cdot \sigma \cdot \frac{N_{\mathrm{O}_{3}}}{\left(1+\frac{E_{b}}{N_{\mathrm{O}_{3}} h c v_{b}}\right) \cdot\left(1+\frac{E_{a}}{2 N_{\mathrm{O}_{3}} h c v_{a}}\right)^{3}} \cdot w
$$

To meet the features of the experimental findings it is sufficient to take into account the well-known ${ }^{26}$ laser active transitions from the ground to the first excited level of the ozone $v_{3}$-vibration. The corresponding number densities of the ro-vibrational sublevels are expressed in the usual way by a product of contributions from a rigid rotor and three independent harmonic oscillators $\left(v_{1}, v_{2}, v_{3}\right){ }^{27}$ The vibrational portion of the difference in number density that governs the absorption process can be expressed in terms of the two harmonic oscillators $v_{a}$ and $v_{b}$ since $v_{1} \approx v_{3} \approx v_{a}$ and 
$v_{2}=v_{b}$, leading via (3.10) to the fraction in Eq. (3.13). The rotational part of this difference $\exp \left[-h c E_{0 j} / k T\right] / Q_{\text {rot }}$ is included in the term $\sigma$

$$
\sigma=\sum_{j} \frac{\sigma_{j} \cdot \exp \left[-\frac{h c E_{0 j}}{k T}\right] / Q_{\mathrm{rot}}(T)}{1+\frac{\sigma_{j} \cdot w}{h v}\left(\frac{1}{g_{0 j}}+\frac{1}{g_{1 j}}\right) \tau_{\mathrm{rot}}(T)}
$$

being the summation over the contributions from individual ro-vibrational transitions $j$. Since the ozone rotation is subsumed under the bath, the occupation of the lower rotational level at $E_{0 j}\left[\mathrm{~cm}^{-1}\right]$ is governed by the temperature $T . Q_{\text {rot }}$ is the classical partition function of rotation ${ }^{27}$

$$
Q_{\mathrm{rot}}(T)=\frac{1}{2}\left[\frac{\pi}{A B C}\left(\frac{k T}{h c}\right)^{3}\right]^{1 / 2}
$$

with $A, B, C$ the rotational constants of ozone.

The denominator in Eq. (3.14) reflects the rotational bottleneck, i.e. the saturation of the rate of vibrational excitation because of lagging rotational relaxation between the laser-active and the remaining ro-vibrational states. Using the rotational degeneracies $g_{0 j}=2 J_{0 j}+1$ and $g_{1 j}=2 J_{1 j}+1$ in the lower and upper vibrational level, it is assumed that each transition $j$ can be looked upon without interference by the remaining transitions ${ }^{28,29}$ Data for the rotational constants $A, B$, and $C$, for the rotational energy $E_{0 j}$, and for the rotational quantum numbers are taken from ${ }^{26}$.

The characteristic time for the rotational relaxation of an ozone molecule colliding with the molecules of an $\mathrm{O}_{2} / \mathrm{O}_{3}$-mixture is given by the ratio of the average number $Z_{\mathrm{rot}}$ of gas kinetic collisions leading to relaxation and the common expression for the gas kinetic collision frequency: ${ }^{18}$

$$
\tau_{\text {rot }}(T)=Z_{\text {rot }} /\left\{\left(K_{\mathrm{O}_{2}} \cdot p_{\mathrm{O}_{2}}+K_{\mathrm{O}_{3}} \cdot p_{\mathrm{O}_{3}}\right) \cdot T^{1 / 2}\right\}
$$

The molecular data of the collision complex $\mathrm{O}_{3} / \mathrm{O}_{3}$ and $\mathrm{O}_{3} / \mathrm{O}_{2}{ }^{30,31}$ are combined in the coefficients $K_{\mathrm{O} 2}$ and $K_{\mathrm{O} 3}$, respectively.

Since the pressure of the gas mixtures ranges from 160 to 1200 mbar, the cross section of an individual transition $j$ at the laser wave number $v$ is governed by a Lorentzian line shape function: ${ }^{32}$

$$
\sigma_{j}=\frac{F_{j} \cdot b_{D}(T)}{\pi\left\{\left(v_{j}-v\right)^{2}+b_{D}^{2}(T)\right\}}
$$

The spectral data, i.e. the line positions $v_{j}$ and the line strengths $S_{j}$ contributing to the coefficients $F_{j}$ are compiled in ${ }^{26}$. To meet experimental findings for the pressure dependence of the $\mathrm{O}_{3}$-absorbance in the low intensity limit ${ }^{33}$ these data were critically revised. It turns out that $F_{j}$ has to be scaled by a constant factor $f_{c}$ chosen the higher the lower the absorbance at the respective laser wavelength is. 
Considering the totality of lines from ${ }^{26}$ between 1040.27 and $1051.42 \mathrm{~cm}^{-1}$ in the region of the 9P18,20, and 22 laser emission and additionally stronger lines with $S_{j}>$ $12 \cdot 10^{-21} \mathrm{~cm} / \mathrm{molec}$. between 1031.45 and $1057.16 \mathrm{~cm}^{-1}$, the scale factor takes the value $f_{c}=1.16$ for the 9P18-line. The calculated pressure dependence of the absorption coefficient shows reasonable agreement with the findings ${ }^{33}$. This is also the case for the 9P20-line if one allows for a slight pressure-dependent line shift, i.e. the replacement of $v_{j}$ by $v_{j}-C_{j} b_{D},{ }^{34}$ for the two $\mathrm{O}_{3}$-transitions adjacent to $v$ with an empirical value of $C_{j}=0.6\left(f_{c}=1.23\right)$. However, at the 9P22-emission, a certain imponderability can be realized. There is only a good agreement in the case of a pressure-independent line shift (replacement of $v_{j}$ by $v_{j}-0.07 \mathrm{~cm}^{-1}$ for the two adjacent transitions). If one discards this non-physical situation one has to accept a pressure-dependent scaling factor in order to meet the experimental findings in the low intensity limit of the present investigation (see below). In this case the values for $\left(p_{\text {mixt }}[\mathrm{mbar}], f_{c}\right)$ range from $(160,2.60),(340,2.35),(690,2.02)$ to $(1200,1.67)$.

The line width $b_{D}$ depends on the translational temperature $T$ and on the pressure $p_{\mathrm{O}_{2}}$ and $p_{\mathrm{O}_{3}}$ of the oxygen and the ozone component, respectively ${ }^{34}$ :

$$
b_{D}(T)=\left(p_{\mathrm{O}_{2}} \cdot b_{\mathrm{O}_{2}}+p_{\mathrm{O}_{3}} \cdot b_{\mathrm{O}_{3}}\right) \cdot(300 / T)^{n}
$$

Since data on the line widths $b_{\mathrm{O}_{2}}$ and $b_{\mathrm{O}_{3}}$ of the individual $j$ for both types of collision partners and also data for the exponent $n$ are still incomplete and contradic-

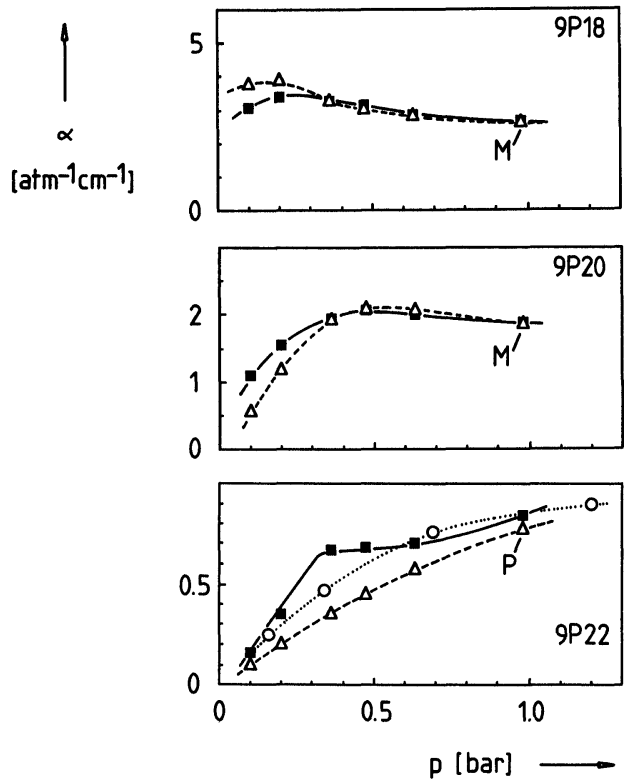

Figure 3 Low-intensity decadic absorption coefficient of $\mathrm{O}_{3}$ in the air of pressure $p$ at $T=300 \mathrm{~K}$ for the laser lines 9P18, 20, 22. $\square,-$ experimental data of Menzies; ${ }^{33} \Delta,-\ldots$ - prediction of the model with constant scale factors $f_{c}$ after adaptation to Menzies ${ }^{33}$ (" $M$ ") and Patty et al. ${ }^{40}$ (" $P$ "); $\circ, \ldots \ldots$ prediction of the model for the 9P22-line from adaptation to the low-intensity region of the present investigation. 
tory to some extent ${ }^{31,35-39}$ it is sufficient to assume average values $b_{\mathrm{O}_{2}}=0.057 \mathrm{~atm}^{-1}$ $\mathrm{cm}^{-1}$ and $b_{\mathrm{O}_{3}}=0.120 \mathrm{~atm}^{-1} \mathrm{~cm}^{-1}$ and a plausible theoretical prediction $n=0.7$. $^{34}$

The details presented so far can be realized in Figure 3, which shows the dependence of the ozone absorption coefficient on the pressure of added air as measured by Menzies ${ }^{33}$ (straight line) compared to the prediction of the model (dashed line) with the average air-broadened linewidth $b_{\text {air }}=0.068 \mathrm{~atm}^{-1} \mathrm{~cm}^{-1} \cdot{ }^{31}$ For the 9P18- and 20-laser line the model was scaled up to the results of Menzies at $p$ $=0.98$ bar (" $M$ "), leading to overall agreement. For the 9P22-line, however, this is not the case. Figure 3 shows the diverging results of Menzies (straight line), of the model with a constant scale factor $f_{c}=1.67$ adatped to Patty et al. ${ }^{40}$ at $p=1$ bar (" $P$," dashed line) and of the model with the varying scale factors given above that were determined from the low intensity limit of the present investigation (dotted line). The inaccuracy of the data and the simplifications of the model are obviously of greater importance in the case of the 9P22-wavelength, which is situated in the region between stronger ozone transitions, c.f. Figure 4.

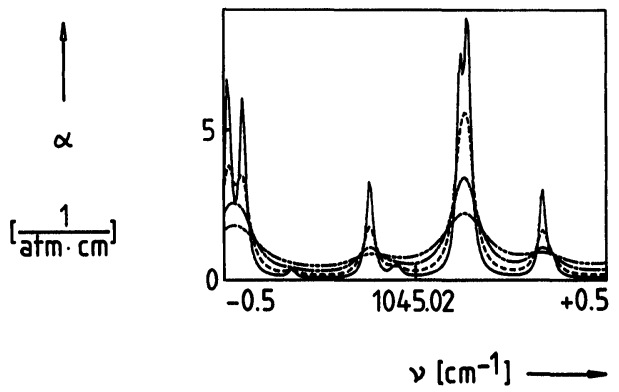

Figure 4 Pressure broadened $\mathrm{O}_{3}$ spectrum ranging from $-0.5 \mathrm{~cm}^{-1}$ to $+0.5 \mathrm{~cm}^{-1}$ around the 9P22-laser line at $1045.02 \mathrm{~cm}^{-1}$ at $T=300 \mathrm{~K}$ according to the model; $17 \mathrm{mbar} \mathrm{O}_{3}$ in $\mathrm{O}_{2} / \mathrm{O}_{3}$ mixtures of the pressures $p_{\text {mixt }}[\mathrm{mbar}]: 160(\longrightarrow), 340(-\cdots), 690(\cdots \cdots \cdots \cdot \cdots), 1200(\cdots \cdots \cdots)$; decadic absorption coefficient.

Finally it should be mentioned that the significance of light absorption in the upper vibrational levels has been investigated in the numerical simulations detailed below. For lack of accurate data, absorption was assumed with a cross section equal to that of the ground state $v=0$ (Eq. (3.14)) for levels up to $v=v_{m}$ that are occupied according to the transient Boltzmann distribution of the model.

\section{SIMULATION AND PROBING OF AN INDIVIDUAL EXCITATION PROCESS}

From the discussion in the preceding chapter it is obvious that the pressure and the composition of the gas mixture determine the excitation and relaxation process in a multiple way. For a constant amount of ozone and increasing oxygen content one has to expect an acceleration of the relaxation (c.f. Eqs. $(3.6,7,16)$ ), an alteration of the 
absorbance because of the pressure broadening (c.f. Eq. (3.18)), and indirect effects on the absorbance for example due to a different history of the transient rovibrational occupation. The appropriate description of the laser excitation and in consequence the laser ignition has therefore to account for the totality of these effects.

This can be done by the numerical simulation of the system of differential equations $(3.2,3,4,11)$ including the space- and time-dependent relations (3.8-10, 12-18) for the energetic and spectroscopic details with respect to the parameters indicated in ch. 3. Discretization of the spatial $z$-coordinate (usually with 25 equi-distant grid points for the cell length) leads to a system of ordinary differential/ algebraic equations that can be solved numerically with the computer code DASSL. ${ }^{41}$ The global temporal laser profile $w(0, t)$ (c.f. Eq. (3.12)) at the cell entrance $(z=0)$ was approximated by a polygonal line that can be taken as boundary condition. For a chosen incoming laser energy DASSL calculates the solutions $\left(E_{a}\right.$, $\left.E_{b}, T, w\right)\left(z_{k}, t_{i}\right)$ for every grid point $z_{k}$ and a given sequence of times $t_{i}$. These solutions represent the spatial and temporal evolution of the three types of internal energy expressed as $E_{a}, E_{b}$, and $T$ as well as their interdependency with the laser energy density $w$ that travels through the absorbing gas mixture with the velocity of light $c$.

A number of results can be deduced from the set $\left(E_{a}, E_{b}, T, w\right)\left(z_{k}, t_{i}\right)$ being directly comparable to the experimental findings. As an example, the simulation of the transient UV-intensity on a perpendicular probe beam (c.f. Figure 1) during and after the laser irradiation will be discussed. As mentioned in ch. 3.1, the laser excitation leads to a time varying Boltzmann occupation of the $v_{a}$-oscillator, i.e. the $v_{1}$ - and $v_{3}$-modes of the real molecule. Since mainly the distribution in the $v_{1}$-mode determines the shape of the Hartley-continuum ${ }^{24}$ the excitation and the relaxation of this vibration becomes observable by UV-light absorption. A quantitative description of these processes can be given by means of the relation (2.2) between the UV-absorption coefficient $\alpha_{T}$ and the vibrational temperature $T_{v}$. In the experiment, the average $T_{v}$ in the volume of intersection of the laser and the probe beam can be calculated from the measured $\alpha_{T}$ (Eq. (2.3)), and, vice versa in the simulation, the absorption coefficient $\alpha_{T}$ and consequently the transmitted UV-light intensity can be obtained from the vibrational temperature $T_{\nu}$ which is determined by $E_{a}$ (c.f. Eq. (3.10)), i.e. the solution modelled by the computer code DASSL.

Figure 5 shows the transients resulting from the irradiation of $\mathrm{O}_{2} / \mathrm{O}_{3}$-mixtures of constant ozone ( 17 mbar) but varying total pressure $p_{\text {mixt }}$ with the same fluence $F_{\text {in }}=$ $1 \mathrm{~J} / \mathrm{cm}^{2}$. Obviously, the global course of the experimental and simulated traces is comparable. Minor differences are to be expected because these are single shot experiments and because of the approach of the radial laser profile by a rectangular profile (c.f. ch. 3.1). The agreement is only attained with the assumption that laser radiation is absorbed in higher vibrational levels up to $v_{m}=2$ (c.f. ch. 3.2) and that the rotational relaxation related to rotational bottleneck is very efficient $\left(Z_{\text {rot }}=\right.$ 1.25). Nevertheless, the significance of increasing pressure for the acceleration of the vibrational relaxation and for the increase of the absorbance due to pressure broadening (c.f. Figure 3,4) is evident. 

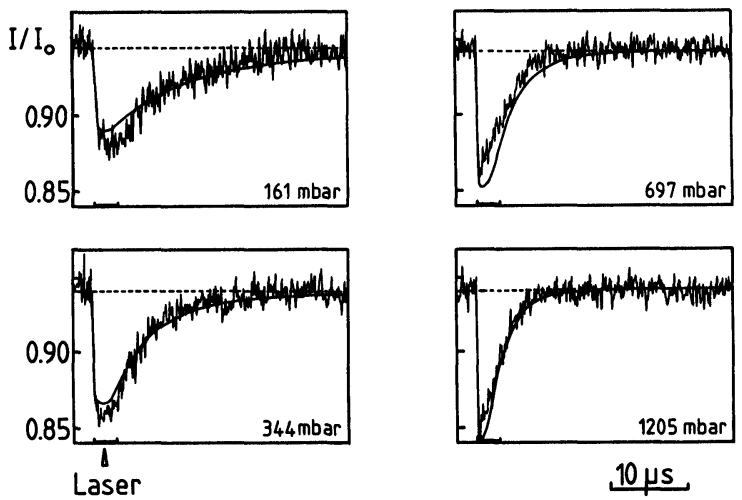

Figure 5 IR-UV-double resonance experiment; UV-transmittance $(\lambda=312.6 \mathrm{~nm})$ on the perpendicular probe beam in the image plane (c.f. Figure 1); irradiation of $17 \mathrm{mbar}_{3}$ in $\mathrm{O}_{2} / \mathrm{O}_{3}$-mixtures of varying total pressure with $F_{\text {in }}=1 \mathrm{~J} / \mathrm{cm}^{2}$ (9P22-line); experiment and simulation (smooth curve; $v_{m}=2, Z_{\text {rot }}=1.25$ ).

From the inspection of transients like those in Figure 5 it follows that the relaxation is slower than the duration of the laser pulse (marked in Figure 5) for pressures up to $p_{\text {mixt }} \approx 340$ mbar. In this case, the absorbed energy density is completely present in the $v_{a}$-oscillator even after passage of the laser pulse and it defines the maximum vibrational temperature $T_{m}$ reached in the oscillator via the Einstein relation (3.10). At higher pressures, the onset of the relaxation during the laser irradiation is obvious and one has to expect a repeated excitation/relaxation cycle, with a certain probability. The vibrational temperature $T_{E}$ calculated from the absorbed energy density should therefore exceed the maximum vibrational temperature $T_{m}$ actually reached in the oscillator. These two different situations can be distinguished experimentally since the UV-transmittance at the minimum of the transients gives directly the maximum vibrational temperature $T_{m}$ from Eq. $(2.2,3)$. The corresponding results are presented in ch. 5.2.

\section{5. $\mathrm{CO}_{2}$-LASER EXCITATION OF OZONE}

\subsection{Pressure and Fluence Dependence of the Absorption Coefficient}

Besides the pressure and the composition of the gas mixture, the power of the laser radiation is of particular significance for the excitation process. Since in this investigaiton the power may reach $10 \mathrm{MW}$, one has to expect a strong saturation of the laser-active transitions in the ozone molecules. In the language of the present model this means that the $v_{a}$-oscillator becomes highly excited, leading to a considerable occupation of the upper levels according to the transient vibrational temperature. For a constant laser profile $f(t)$ (c.f. Eq. (3.12)), the increase over a certain 
fluence should therefore result in a reduction of the absorption coefficient at the laser wavelength. The (decadic) absorption coefficient is given by

$$
\alpha=\frac{1}{p_{\mathrm{O}_{3}} \cdot l} \log \frac{E_{\text {in }}}{E_{\text {out }}}
$$

where $E_{\text {in }}$ is the incoming, $E_{\text {out }}$ the outgoing pulse energy and $l$ the cell length.

The corresponding measurements were carried out in $\mathrm{O}_{2} / \mathrm{O}_{3}$-mixtures of $17 \mathrm{mbar}$ ozone and total pressures $p_{\text {mixt }}=160,340,690$, and 1200 mbar with fluences ranging from $0.01 \mathrm{~J} / \mathrm{cm}^{2}$ up to nearly $2.00 \mathrm{~J} / \mathrm{cm}^{2}$. Normally the pulses were "short" ones with a leading spike of $130 \mathrm{nsec}$ half-width and a reduced $\mathrm{N}_{2}$-tail but in some cases also "long" ones with a spike of $160 \mathrm{nsec}$ and an enhanced $\mathrm{N}_{2}$-tail. The spike region (up to $500 \mathrm{nsec}$ ) of the first ones usually contained roughly $80 \%$ of the pulse energy, that of the latter ones $68 \% .^{7}$

The data analysis follows a well established technique for experiments on nonlinear laser absorption. ${ }^{42,43}$ The measured absorption coefficient $\alpha$, Eq. (5.1), is assigned to a reduced fluence $\bar{F}_{\text {in }}$ in order to give approximately a local view of cause (the fluence $\bar{F}_{\text {in }}$ ) and effect (the measured $\alpha$ ). Therefore the fluence is weighted by an estimated spatial laser profile being Gaussian in the radial coordinate with an average parameter $n=8$ for the whole cell length $l$ (c.f. Eq. (2.1)), and exponential in the longitudinal direction. This procedure gives

$$
\bar{F}_{\text {in }}=\frac{E_{\text {in }}}{\pi a^{2}} \cdot \frac{1-10^{-\alpha \cdot p \mathrm{O}_{3} \cdot 1}}{2^{1 / 4} \Gamma(5 / 4) \cdot \alpha \cdot p_{\mathrm{O}_{3}} \cdot l \cdot \ln 10}
$$

with $a$ the $1 / e$-radius of the laser profile and $\alpha$ from Eq. (5.1). It should be mentioned that $\bar{F}_{\text {in }}$ differs only negligibly from $F_{\text {in }}=E_{\text {in }} /\left(\pi a^{2}\right)$ because of the minor absorption in the experiments of this work.

The experimental results are shown in Figure 6. Starting with negligible effects at low fluences, saturation is observed for increasing laser irradiation, leading to a reduction of the absorption coefficient by a factor of up to 10 in the case of the strongly absorbed 9P18-laser line. The results are consistent with low intensity data of Menzies ${ }^{33}$ and Patty et al. ${ }^{40}$ which are marked in Figure 6 by " $M$ " and " $P$ " for ozone in air at atmospheric pressure. " $P$ " denotes the range of values obtained by Patty $e t$ al. and other workers cited in their paper, reflecting the well-known uncertainty of ozone results.

The effect of the collision partners is obviously the same for the three lines investigated, resulting in stronger absorption for increasing pressure of the gas mixture. The extent, however, is different for the three lines, apparently reflecting the position of the laser wavelength with respect to the ozone transitions. In the case of the 9P18-line, the emission is situated on the wing of an ozone transition where, according to the pressure broadening, the variation of the absorption coefficient is less pronounced than in the case of the 9P22-line, for example, where the laser emission is found exactly between two ozone transitions, c.f. Figure 4.

The effect of the "long" laser pulse compared to the usually investigated "short" one is exemplified for $p_{\text {mixt }}=340$ bar in the case of the 9P22-line, c.f. Figure 6. It 


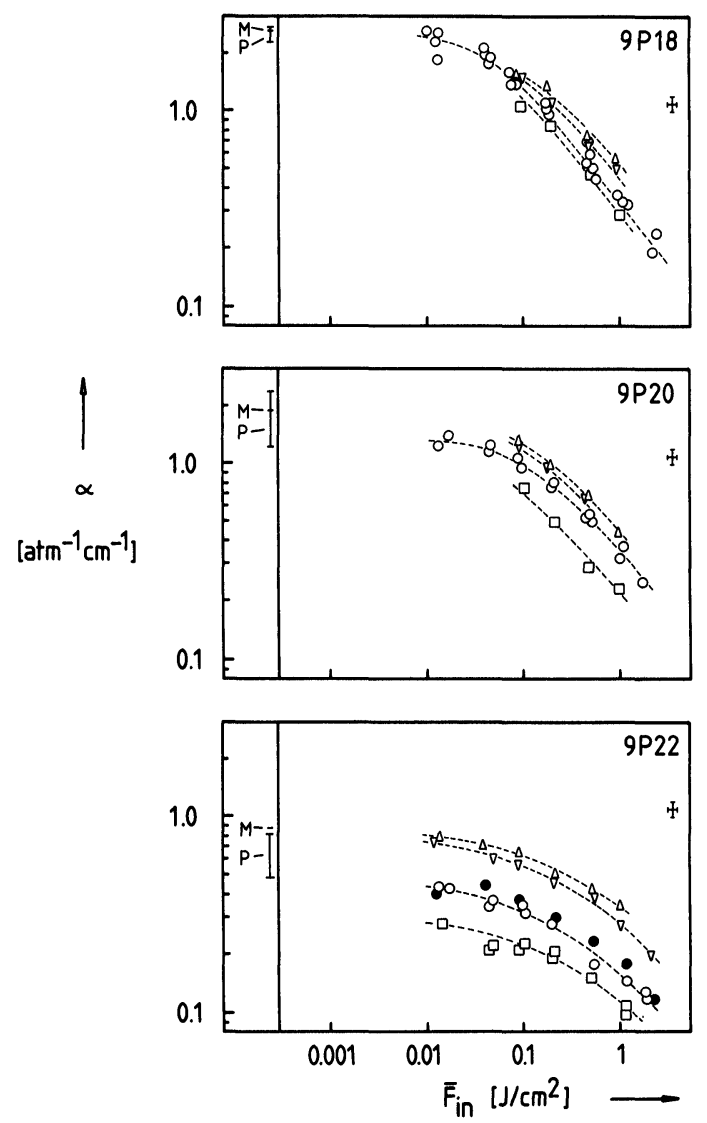

Figure 6 Decadic absorption coefficient for laser pulse absorption in $17 \mathrm{mbar} \mathrm{O}_{3}$; pressure of the $\mathrm{O}_{2} / \mathrm{O}_{3}$ mixtures $p_{\text {mixt }}[\mathrm{mbar}]: 160(\square), 340(\circ \bullet), 690(\nabla), 1200(\Delta)$; laser lines: 9P18, 20 and 22; open symbols: short laser pulse, closed symbols: long pulse; low-intensity results for $\mathrm{O}_{3}$ in 1 bar of air from Menzies ${ }^{33}$ (" $M$ ") and Patty et al..$^{40}$ ("P").

turns out that the rise of the absorption for the long pulse is not significant compared to the error limits. Therefore the pulse profile has to be lengthened to a greater extent than in this investigation in order to reduce the saturation effects.

The recorded data on the absorbed laser energy can be evaluated as an absorption coefficient (Eq. (5.1)) but also as the average number $N_{\text {abs }}$ of laser photons absorbed per $\mathrm{O}_{3}$ molecule. The dependencies are, in summary, an increasing number of absorbed photons with increasing fluence and pressure. For the 9P22-line, as an example, $N_{\text {abs }}$ ranges from 0.05 to roughly 1.6 for the fluence raised from 0.02 to 1.1 $J / \mathrm{cm}^{2}$ at the pressure $p_{\text {mixt }}=1200$ mbar. For $p_{\text {mixt }}=160 \mathrm{mbar}, N_{\mathrm{abs}}$ amounts, however, to only 0.5 at $1.1 \mathrm{~J} / \mathrm{cm}^{2}$. The order of magnitude of these values compares quite well with findings for the highly absorbed 9P30-line obtained for total pressures up to 70 mbar. ${ }^{44,45}$. The relatively high number of absorbed photons in the present 
investigation is obviously a consequence of the vibrational multi-level system, allowing additional absorption in upper levels and also of the fast intramode redistribution effecting, to a certain extent, repeated absorption in the lower levels.

The fluence dependence of the laser excitation has also been modeled with the computer code described in ch. 4 . The results will be given in the next section in terms of the maximum vibrational temperature reached in the $v_{\mathrm{a}}$-oscillator.

\subsection{Maximum Vibrational Temperature Resulting from Laser Excitation}

According to the model and the findings in ch. 4, the maximum vibrational temperature $T_{m}$ in the laser pumped ozone oscillator is a key parameter for the description of the excitation process. Because of relatively slow intermode relaxation at pressures $p_{\text {mixt }} \leqslant 340 \mathrm{mbar}$, the temperature $T_{E}$, calculated from the absorbed energy density $E_{\text {abs }}$ should equal $T_{m}$, which can be measured independently via the minimum of the transient UV-signals. At pressures $p_{\text {mixt }}>340 \mathrm{mbar}, T_{E}$ is expected to exceed the $T_{m}$-value actually reached reflecting the onset of the relaxation still during the laser pulse.

$T_{E}$ is related to $E_{\text {abs }}$ via (c.f. Eq. (3.10)):

$$
T_{E}=\frac{h c v_{a}}{k \cdot \ln \left\{1+2 N_{\mathrm{O}_{3}} h c v_{a} /\left(E_{a 0}+E_{\mathrm{abs}}\right)\right\}}
$$

where $E_{a 0}$ is the energy content in the $v_{a}$-oscillator at room temperature before the irradiation. As an example, Figure 7 shows the fluence dependence of $T_{E}$ (filled symbols) for the 9P22-line at the pressure $p_{\text {mixt }}=340$ mbar.

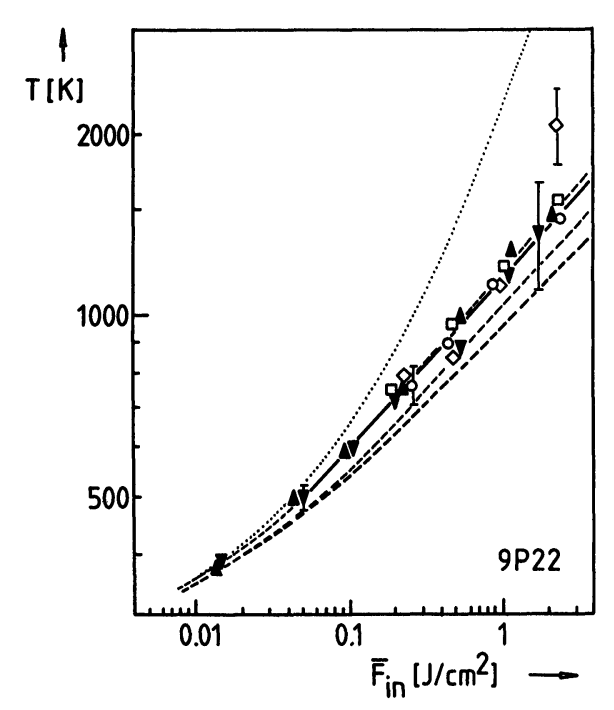

Figure 7 Temperatures in the laser pumped $\mathrm{O}_{3}$ oscillator $v_{a}$; 9P22-laser line; 17 mbar $\mathrm{O}_{3}$ in an $\mathrm{O}_{2} / \mathrm{O}_{3}$-mixture of $p_{\text {mixt }}=340$ mbar; temperature before irradiation: $T=300 \mathrm{~K}$.

(1) Temperature $T_{E}$ from absorbed laser energy density (see text): (a) experiment; short pulse $(\boldsymbol{\nabla})$, long pulse $(\boldsymbol{\Lambda}),-$ sion. (b) simulation; - . - . - ; curves in increasing order for $\left(Z_{\text {rot }}, v_{m}\right)=(5.00,0),(5.00,2)$, $(1.25,2)$. (c) Simulation; . . . . . . ; for comparison, with constant room temperature occupation $\left(v_{m}=2\right)$.

(2) Maximum vibrational temperature $T_{m}$ from UV-transients: long pulse; $\lambda_{\mathrm{UV}}[\mathrm{nm}]=296.7(\diamond)$, 302.2 (), 312.6 (०). 
In this case, but also for the other pressures and laser lines investigated $T_{E}\left(\bar{F}_{\text {in }}\right)$ can be approximated by a linear dependency in the logarithmic representation provided $T_{E}$ exceeds about $400 \mathrm{~K}$. In Figure 7 this situation is met for $\bar{F}_{\text {in }} \gtrsim 0.05 \mathrm{~J} / \mathrm{cm}^{2}$, whereas for the 9P18- and the 9P20-line it is observed in the whole fluence range due to the stronger excitation. The exponents $m=m_{E}$ of the corresponding exponential expression

$$
T=\text { const } \cdot \bar{F}_{\text {in }}^{m}
$$

can be determined from the linear regression of the experimental results shown in Fig. 7 as a straight line. The values are given approximately by $m_{E}=0.3$ for $p_{\text {mixt }}=$ 160 and 340 mbar but increase considerably for $p_{\text {mixt }}=690$ and $1200 \mathrm{mbar}$, c.f. Table 1.

Table 1 Pressure dependence of the exponent $m$ in Eq. (5.4) for the 9P22-laser line.

\begin{tabular}{cll}
\hline$p_{\text {mixt }}$ [mbar] & $\begin{array}{l}m=m_{E}, \text { from absorbed } \\
\text { laser energy density }\end{array}$ & $\begin{array}{l}m=m_{U V}, \text { from } \\
\text { UV-transients }\end{array}$ \\
\hline 160 & $0.29 \pm 0.06$ & $0.29 \pm 0.02$ \\
340 & $0.28 \pm 0.06$ & $0.30 \pm 0.01$ \\
690 & $0.36 \pm 0.05$ & $0.29 \pm 0.02$ \\
1,200 & $0.38 \pm 0.05$ & $0.28 \pm 0.03$ \\
\hline
\end{tabular}

The simulation was carried out for the same fluences, gas mixtures and laser lines as the experiments, leading to temperatures $T_{E}$ by means of Eq. (5.3) via the calculated absorbed energy density $E_{\text {abs }}$. The results are given by the dashed curves in Figure 7. According to ch. 3.2, scale factors $f_{c}$ were introduced for the adaptation of the simulated to the experimental values at $F_{\text {in }} \approx 0.01 \mathrm{~J} / \mathrm{cm}^{2}$ in the case of the 9P22-line. This procedure is justified since at the lower limit of the investigated fluence range the occupation of the upper vibrational levels and therefore the saturation is still negligible, c.f. ch. 5.1. Furthermore, this agreement is automatically attained, even for the strongly absorbed 9P18- and 9P20-lines, the scale factors of which were determined independently by comparison with the low intensity data of Menzies, ${ }^{33}$ c.f. ch. 3.2 .

The dashed curves represent runs of the simulation code with a different choice of parameters for the rotational relaxation $\left(Z_{\text {rot }}\right)$ and the maximum vibrational level $v_{m}$ that contributes to the absorption. The lowest curve covers reliable values from the literature, $Z_{\text {rot }}=5$ being typical for a lot of small molecules, ${ }^{18}$ and $v_{m}=0$ accounting only for the well-known ${ }^{26}$ ground state absorption of ozone. If one allows for the absorption in the vibrational levels up to $v_{m}=2\left(Z_{\text {rot }}=5\right)$, a considerable increase of the temperature $T_{E}$ is calculated, especially for $\bar{F}_{\text {in }}>0.1 \mathrm{~J} / \mathrm{cm}^{2}$, as shown by the adjacent curve. The inclusion of even higher absorbing levels results only in a negligible increase because of their minimal occupation, at least if the same cross section (that of the ground state) is assumed for all these states, c.f. ch. 3.2.

Also the second parameter, the number of collisions $Z_{\text {rot }}$ leading to rotational 
relaxation, affects the extent of the excitation. If a very efficient relaxation is assumed $\left(Z_{\text {rot }}=1.25\right)$, the simulated $T_{E}$-dependency meets exactly the experimental results provided $v_{m}=2$, c.f. the uppermost curve. This is also observed for the same set of parameters in the case of the 9P18-laser line ( $\left.p_{\text {mixt }}=340 \mathrm{mbar}\right)$. The experimental results for the $9 \mathrm{P} 20$-line $\left(p_{\text {mixt }}=340 \mathrm{mbar}\right)$, however, considerably exceed the simulated ones, reflecting possibly a strong absorption in the upper vibrational levels.

Apart from the difference between the experimental and the various simulated results, the modeled dependency shows the same trend for $T_{E}>400 \mathrm{~K}$, i.e. a roughly linear dependence with a comparable slope in the logarithmic representation. Exactly this trend is characteristic for the saturated laser excitation in the vibrational ladder under the collision regime, at least in the investigated fluence range. This can be concluded by comparison with the dotted curve in Figure 7, which was simulated with exclusion of any saturation, i.e. the assumption of a constant room temperature occupation of the ro-vibrational states.

Finally, the results for the maximum vibrational temperatures $T_{m}$ are presented which were obtained in separate experiments from the minimum of the UVtransients. $T_{m}$ is determined using the Sulzer-Wieland expression (2.2) that is verified experimentally only in the temperature range $300 \mathrm{~K} \leq T \leq 1050 \mathrm{~K}^{13}$ According to the present investigation, the extension of this range seems to be

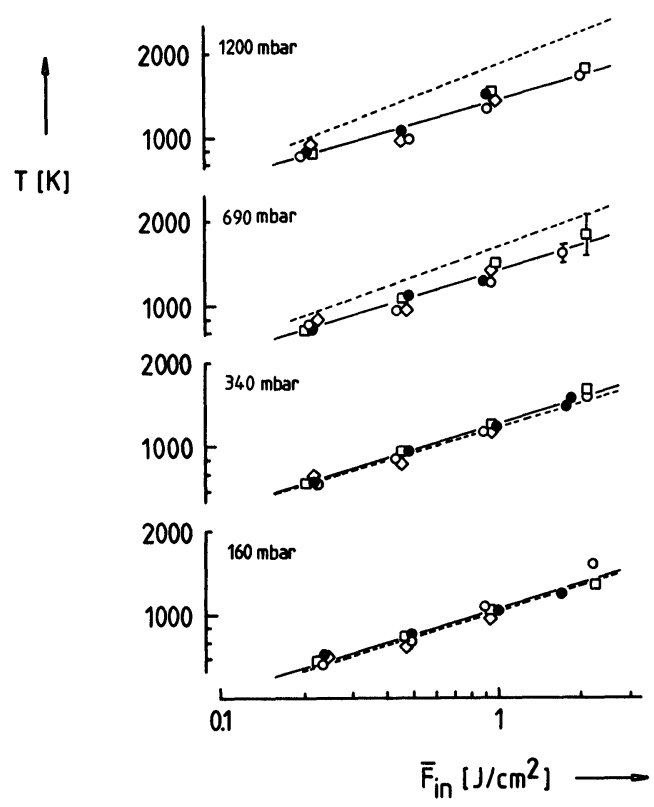

Figure 8 Temperatures in the laser pumped $\mathrm{O}_{3}$-oscillator $v_{a}$; 9P22-laser line; 17 mbar $\mathrm{O}_{3}$ in $\mathrm{O}_{2} / \mathrm{O}_{3}$ mixtures of various pressures. (1) Temperature $T_{E}$ from absorbed laser energy density: - . . . . - linear regression of the individual results not shown. (2) Maximum vibrational temperature $T_{m}$ from UVtransients: $\lambda_{u v}[\mathrm{~nm}]=296.7(\diamond), 302.2(\square), 312.6(\diamond \bullet)$, open: long pulse, closed: short pulse; linear regression. 
warranted up to $1300 \mathrm{~K}$ for the wavelengths $\lambda=296.7,302.2,312.6 \mathrm{~nm}$ and even up to $1500 \mathrm{~K}$ for $\lambda 302.2,312.6 \mathrm{~nm}$, since the temperatures determined using Eq. (2.2) for the wavelengths indicated are consistent in the respective range as can be deduced by inspection of Figures 7 and 8 .

The maximum vibrational temperatures $T_{m}$ for $p_{\text {mixt }}=340$ mbar are marked by open symbols in Figure 7. Obviously, they agree with the temperatures $T_{E}$ from the absorbed energy density as is also observed for the lower total pressure $p_{\text {mixt }}=160$ mbar, c.f. Figure 8 . In this figure the results for the various pressures $p_{\text {mixt }}$ are presented and, for comparison, the corresponding linear regressions of the $T_{E}$-data (dashed lines). For both the higher pressures the $T_{E}$-values exceed the $T_{m}$ temperatures and show furthermore a steeper slope.

Actually, the exponent $m=m_{\mathrm{UV}}$ for the UV-results (Eq. (5.4)) is roughly given by $m=0.3$ in the whole pressure range, c.f. Table 1 . The maximum vibrational temperature reached in the laser pumped oscillator is therefore determined by the simple exponential expression (5.4) without regard to the course of the relaxation process.

\section{CONCLUSIONS}

The features of the transient vibrational $\mathrm{O}_{3}$-excitation as well as the fluence dependence of this excitation are reproduced fairly well by a simplified model based upon the relaxation theory of harmonic oscillators (SSH-theory). ${ }^{11,12}$ The excitation of $\mathrm{O}_{2} / \mathrm{O}_{3}$-mixtures with higher ozone content, i.e. of ignitable mixtures, can therefore be simulated principally with regard to the gradient of the absorbed laser energy being more pronounced than in the present investigation. In any case these are prerequisites for an "ab initio" simulation of the spatial and temporal evolution of an ignition process that is initiated by the absorption of pulsed $\mathrm{CO}_{2}$-laser radiation.

However, for a comprehensive modelling, more accurate data should be available for several important parameters such as the rate constants for rotational relaxation, which govern the extent of excitation via the rotational bottleneck, or high resolution spectral data in order to describe the absorption of laser light in the excited vibrational levels.

\section{Acknowledgement}

The authors thank Dr. L. Petzold for providing the computer code DASSL and Dr. U. Maas for assistance in the computer simulations. The financial support of the "Deutsche Forschungsgemeinschaft" (SFB 123, Stochastische Mathematische Modelle) is gratefully acknowledged.

\section{References}

1. B. Raffel, J. Warnatz, H. Wolff, and J. Wolfrum, Proc. 6th Annual Meeting IEA Committee on Conservation in Comb., Sandia, Livermore, p. 112 (1984).

2. B. Raffel, J. Warnatz, H. Wolff, J. Wolfrum and R. J. Kee, Progr. Astronaut. Aeronaut. 105(II), 355 (1986). 
3. B. Raffel, J. Warnatz and J. Wolfrum, Appl. Phys. B37, 189 (1986).

4. B. Raffel and J. Wolfrum, Ber. Bunsenges. Phys. Chem. 90, 997 (1986).

5. U. Maas, B. Raffel, J. Wolfrum and J. Warnatz, 21st Symp. (Int.) on Comb., 1869 (1986).

6. B. Raffel and J. Wolfrum, Z. Phys. Chem. N. F. 161, 43 (1989).

7. B. Raffel, Dissertation Thesis, University of Heidelberg, 1988.

8. U. Maas, Dissertation Thesis, University of Heidelberg, 1988.

9. J. Warnatz, Ber. Bunsenges. Phys. Chem. 82, 193 (1978).

10. J. M. Heimerl and T. P. Coffee, Comb. Flame 39, 301 (1980).

11. R. N. Schwartz, Z. I. Slawsky and K. F. Hertzfeld, J. Chem. Phys. 20, 1591 (1952).

12. D. I. Rosen and T. A. Cool, J. Chem. Phys. 62, 466 (1975).

13. D. C. Astholz, A. E. Croce and J. Troe, J. Phys. Chem. 86, 696 (1982).

14. D. J. McCaa and J. H. Shaw, J. Mol. Spectrosc. 25, 374 (1968).

15. S. M. Adler-Golden, E. L. Schweitzer and J. I. Steinfeld, J. Chem. Phys. 76, 2201 (1982).

16. S. M. Adler-Golden and J. I. Steinfeld, Chem. Phys. Lett. 76, 479 (1980).

17. T. G. Kreutz, J. A. O'Neill and G. W. Flynn, J. Phys. Chem. 91, 5540 (1987).

18. J. T. Yardley, Introduction to Molecular Energy Transfer (Academic Press, New York, 1980).

19. O. P. Judd, J. Chem. Phys. 71, 4515 (1979).

20. M. Born and E. Wolf,. Principles of Optics (Pergamon Press, Oxford, 1986).

21. A. Burcat, chap. 8 in: W. C. Gardiner (ed.), Combustion Chemistry (Springer-Verlag, Berlin, 1984).

22. D. I. Rosen and T. A. Cool, J. Chem. Phys. 59, 6097 (1973).

23. I. C. McDade and W. D. McGrath, Chem. Phys. Lett. 72, 432 (1980).

24. J. A. Joens, J. B. Burkholder and E. J. Bair, J. Chem. Phys. 76, 5902 (1982).

25. J. D. Jackson, Classical Electrodynamics (Wiley \& Sons, New York, 1975).

26. A. Barbe, C. Secroun, P. Jouve, N. Monnanteuil, J. C. Depannemaecker, B. Duterage, J. Bellet and P. Pinson, J. Mol. Spectrosc. 64, 343 (1977).

27. G. Herzberg, Molecular Spectra and Molecular Structure (van Nostrand Reinhold Co., New York, 1945).

28. V. S. Letokhov and A. A. Makarov, Sov. Phys. JETP 36, 1091 (1973).

29. V. N. Bagratashvili, V. S. Letokhov, A. A. Makarov and E. A. Ryabov, Laser Chem. 4, 1 (1983).

30. J. Warnatz, Ber. Bunsenges. Phys. Chem. 82, 193 (1978).

31. R. R. Gamache and R. W. Davies, J. Mol. Spectrosc. 109, 283 (1985).

32. S. S. Penner, Quantitative Molecular Spectroscopy and Gas Emissivities (Addison-Wesley, Reading, 1955).

33. R. T. Menzies, Appl. Opt. 15, 2597 (1976).

34. A. Unsöld, Physik der Sternatmosphären (Springer-Verlag, Berlin, 1955).

35. G. D. T. Tejwani and E. S. Yeung, J. Chem. Phys. 63, 1513 (1975).

36. J. M. Hoell, C. N. Harward, C. H. Bair and B. S. Williams, Opt. Engin. 81, 548 (1982).

37. C. Meunier, P. Marche and A. Barbe, J. Mol. Spectrosc. 95, 271 (1982).

38. R. R. Gamache, J. Mol. Spectrosc. 114, 31 (1985).

39. R. R. Gamache and L. S. Rothman, Appl. Opt. 24, 1651 (1985).

40. R. R. Patty, G. M. Russwurm, W. A. McClenny and D. R. Morgan, Appl. Opt. 13, 2850 (1974).

41. L. R. Petzold, SANDIA-Report SAN 82-8637, SANDIA Nat. Lab. (Livermore, 1982).

42. J. L. Lyman and K. M. Leary, J. Chem. Phys. 69, 1858 (1978).

43. J. L. Lyman, G. P. Quigley and O. P. Judd, p. 9 in C. D. Cantrell (ed.), Multiple-Photon Excitation and Dissociation of Polyatomic Molecules (Springer-Verlag, Berlin, 1986).

44. S. M. Adler-Golden and J. I. Steinfeld, Chem. Phys. Lett. 76, 479 (1980).

45. M. S. Dzhidzhoev, V. K. Popov, V. T. Platonenko and A. V. Chugunov, Sov. J. Quantum Electron. 14, 917 (1984). 